\title{
Anaesthetic Challenges in Thoracic Spine Surgery with Bilateral Pulmonary Bullae: A Case Report with Literature Review
}

\section{Barik AK, Jain G* and Gnaneswaran HH \\ Department of Anaesthesiology, All India Institute of Medical Sciences, India}

*Corresponding author: Gaurav Jain, Associate professor, Department of Anaesthesiology, All India Institute of Medical Sciences, Rishikesh-249203, Uttarakhand, India; Tel: +918808631209; Email: gauravhld@gmail.com

\section{Case Report}

Volume 4 Issue 2

Received Date: June 15, 2019

Published Date: July 17, 2019

\section{Abstract}

Bullae are thin walled, air filled intraparenchymal lung spaces which carries a significant risk of life-threatening complications during the perioperative period. To avoid the risk of rupture, most experts recommend the use of spontaneous ventilation or regional anaesthesia during the intraoperative period. We present the anaesthetic management of an elderly smoker with bilateral pulmonary bullae, poorly controlled diabetes, and hypertension, posted for excision of a thoracic-spine tumour under the prone position, requiring need for general anaesthesia with positive pressure ventilation. This literature review highlights the steps for pre-operative evaluation, anaesthesia planning, precautionary measures, and the protocol to be followed in the event of complications.

Keywords: Extradural tumour; Prone position; Pulmonary bullae; Thoracic surgery

\section{Introduction}

Perioperative management of patient with bilateral pulmonary bullae is a challenging task for the anaesthesiologist. We report the anaesthetic management of an elderly patient posted for excision of an extraduralintramedullary spine tumour with bilateral pulmonary bullae, under the prone position.

\section{Case History}

A 65-year-old male was planned for the laminectomy procedure and subsequent excision of $\mathrm{D}_{4}-\mathrm{D}_{6}$ extraduralintramedullary spine tumour, under a prone position. He had been smoking for 10 years, a known diabetic for 10 years and hypertensive for 2 years; on irregular medications. There was bilaterally diminished air entry on chest auscultation. On chest skiagram, we observed multiple bullae in the middle portion of the right lung and the lower zones of the left lung. The contrast-enhanced computed tomography thorax confirmed multiple bullae in middle and upper lobes of the right lung, and anterolateral-basal segments of left lower and lingual lobe (Figure 1). The largest bullae measured approximately 3.0 x $2.8 \mathrm{~cm}$. There were associated fibrocalcific changes in bilateral mediastinal lymph nodes. A restrictive pattern was observed in the pulmonary function test (PFT). Arterial blood gas (ABG) analysis revealed a partial pressure of oxygen $\left(\mathrm{PaO}_{2}\right)$ of $50 \mathrm{~mm} \mathrm{Hg}$, partial pressure of carbon dioxide $\left(\mathrm{PaCO}_{2}\right)$ of $62 \mathrm{~mm} \mathrm{Hg}$, and partial 


\section{Anaesthesia \& Critical Care Medicine Journal}

oxygen saturation $\left(\mathrm{SpO}_{2}\right)$ of $88 \%$, at room air. The echocardiogram and other laboratory parameters were

unremarkable.

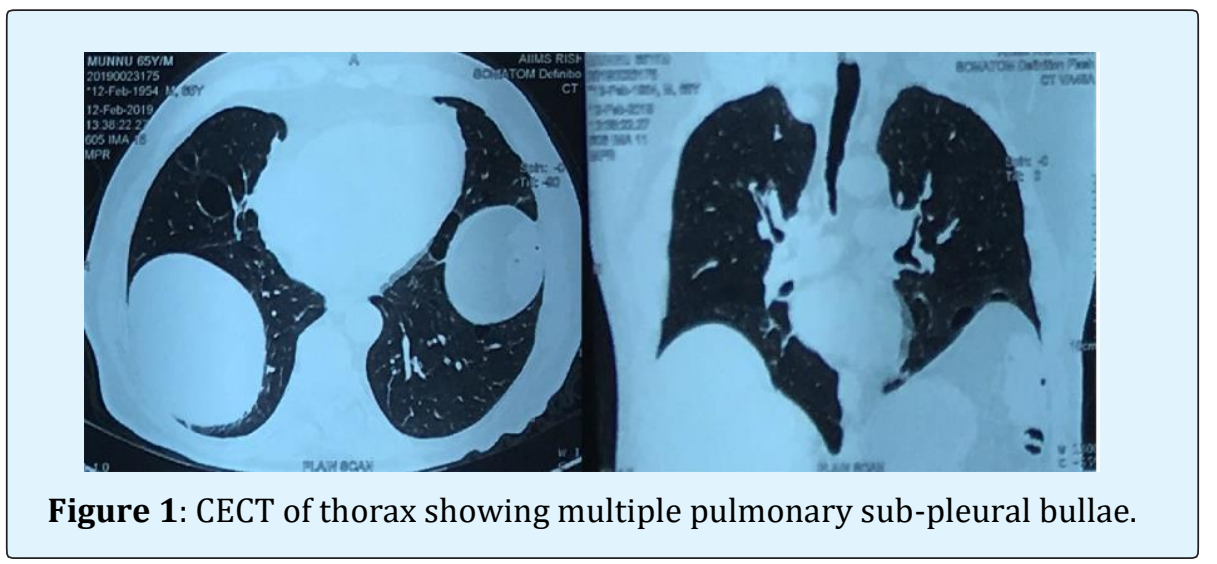

After cessation of smoking, chest physiotherapy, drug optimization, and under a suitable anaesthetic preparation, the patient was shifted to the operation theatre, and a multipara monitor and intravenous (IV) line were secured. Topical airway anaesthesia was achieved by nebulisation with $4 \%$ lignocaine, and airway reflex was blunted with a superior laryngeal block (bilaterally) and trans-tracheal instillation of $2 \%$ lignocaine, respectively. Following preoxygenation via face mask (oxygen at 6 litres/minute), general anaesthesia (GA) was induced with lidocaine $(1.5 \mathrm{mg} / \mathrm{kg}$ IV), propofol (2 mg/kg IV), and fentanyl ( $2 \mu \mathrm{g} / \mathrm{kg} \mathrm{IV}$ ) under gentle manual ventilation by a reservoir bag. Tracheal intubation (Double lumen tube) was assisted with vecuronium $(0.1 \mathrm{mg} / \mathrm{kg}$ IV). Thereafter, mechanical ventilation was started (volume-controlled mode) at a lower tidal volume strategy $(5 \mathrm{ml} / \mathrm{kg})$, and respiratory rate of 12 breaths/minute adjusted to target the end-tidal carbon dioxide $\left(\mathrm{EtCO}_{2}\right)$ levels of $50-60 \mathrm{~mm} \mathrm{Hg}$. The maximum airway pressure limit was kept at $20 \mathrm{~cm} \mathrm{H}_{2} \mathrm{O}$ without any positive end-expiratory pressure (PEEP). The fractional inspired oxygen concentration $\left(\mathrm{FiO}_{2}\right)$ was titrated to maintain the $\mathrm{SpO}_{2}$ between $94-98 \%$. The stethoscopes were attached on the thorax region (one on each side), for intraoperative monitoring of the bilateral air entry into the thorax. An arterial line and an extralarge bore IV cannula were also secured. The patient was shifted on to prone position over the appropriately sized bolsters and head support. The extra cotton paddings were applied below the chest and at other pressure points. The intraoperative anaesthesia was maintained with sevoflurane $(0.5-2 \%)$, air: oxygen mixture $(3: 2)$, fentanyl boluses $(2 \mu \mathrm{g} / \mathrm{kg}$ IV) and vecuronium infusion (0.04 mg/kg/hour IV). The bi-spectral index level was kept in between 40-60 range. The operative procedure lasted for 4 hours, with an approximate blood loss of 300 $\mathrm{ml}$. The blood sugar levels were kept at $120-180 \mathrm{mg} / \mathrm{dl}$ throughout the intraoperative period. The patient was reverted to a supine position at the end of surgery and shifted to the post-operative care unit in an intubated state. The extubation was performed after 1 hour, in a fully-awake state without any complications.

\section{Discussion}

By definition, a pulmonary bulla is described as a cavity larger than $1 \mathrm{~cm}$ in diameter, formed within the attenuated pulmonary parenchyma [1]. Though the underlying mechanism of a bullae formation is uncertain, common contributing factors include the emphysema, chronic smoking, alpha-1 antitrypsin deficiency, fibrotic lung diseases, tuberculosis, sarcoidosis, marfan's syndrome, and chronic inflammation [1]. It is also known to be associated with bronchogenic carcinoma. A bulla can exist as a singular unit, in multiple or the cluster forms. The walls of a bulla are more compliant than the surrounding normal lung tissues and exhibit the physical effects of Laplace's law. It offers lower resistance to inflation, so can inflate further without sufficient deflation [2]. Bullae can be either communicating or the compliant type. If the bulla communicates with the bronchial tree, positive pressure ventilation may cause it to expand or rupture. If the bulla is highly compliant, the tidal volume may be wasted in the form of dead space ventilation $[3,4]$. An enlarging bulla can also lead to compression of the surrounding normal lung tissues and mediastinum or may rupture leading to tension pneumothorax, pneumopericardium, hypoxemia, haemorrhage, venous 


\section{Anaesthesia \& Critical Care Medicine Journal}

gas embolism, infection, haemodynamic collapse and eventual mortality in spite of cardiopulmonary bypass $[4,5]$. Surgery under a prone position poses additional challenges including an increase in upper airway resistance due to pressure of chest rolls, decreased venous return and stroke volume with compensatory increase in heart rate, abdominal compression leading to vena-caval compression further associated with increased surgical bleeding, rise in intracranial pressure due to decreased jugular venous return, and increased risk of accidental extubation [6]. Vigilant monitoring of ventilatory parameters, periodic auscultation of the chest and the availability of chest drainage tube are of paramount importance in detecting and managing any intraoperative complication.

In such patients, a detailed pulmonary evaluation is vital during the pre-operative assessment. The radiological work-up helps in evaluating the exact site, number and size of the bullae. A PFT delineates a restrictive pattern, coherent with the severity of the pulmonary disease, the risk of perioperative complications, and need of postoperative ventilation. A pre-operative $A B G$ analysis helps in evaluating the baseline acid-base status and helps to target the intraoperative ventilatory parameters for the patient ${ }^{7}$. Preoperatively, the patient's respiratory functions should be optimised as far as possible, with chest physiotherapy. The patient should be advised to quit smoking and to continue all other bronchodilator medications. The maximum benefit is obtained if smoking is stopped at least 8 weeks before the surgery. Though, some studies suggest that smoking cessation of fewer than 8 weeks before surgery is associated with increased risk of postoperative complications, we did not observe such outcome [7]. The patient attendants should also be counselled accordingly, regarding the possible complications and the anticipated post-operative course.

Different anaesthetic approaches have been utilized for the patients with bullae undergoing thoracic surgery, keeping in mind the underlying co-morbidities, type of surgery and the duration of the procedure. This includes inhalational anaesthesia with spontaneous ventilation, surgery under conscious sedation, GA with manual or mechanical ventilation or the use of regional anesthesia $[5,8,9]$. The basic rule is to avoid the bullae expansion while preserving adequate ventilation. We engaged GA with mechanical ventilation considering the long duration of surgery and need for prone positioning. To avoid the associated risks, we targeted a protective ventilation strategy with permissive hypercapnia keeping in mind the baseline status of the patient. Due to the associated risks of bullae rupture, positive pressure ventilation was avoided during the induction period, until the patient is intubated $^{5}$. We ventilated the patient via a gentle manoeuvre with a reservoir bag, during this phase. A better intubating condition was achieved by providing local anaesthesia to the upper airway using a topical method and nerve blocks [8].

During the intraoperative period, preventing any coughing and straining during the induction and recovery phase of anaesthesia, avoiding the use of nitrous oxide, ascertaining an adequate depth of the anaesthesia, and optimized analgesia are some of the key principles for minimizing the complications. The best ventilation practice in a patient with bullae involves an air: oxygen mixture, PIP $<20 \mathrm{~cm} \mathrm{H}_{2} \mathrm{O}$, tidal volume of $5-6 \mathrm{ml} / \mathrm{kg}$ of ideal body weight, permissive hypercapnia, slow inspiratory flow rate, decreased inspiratory: expiratory ratio to prevent air trapping (1:4-1:5), and avoidance of PEEP [10]. As $\mathrm{EtCO}_{2}$ may grossly underestimate the arterial $\mathrm{PaCO}_{2}$, so it is advisable to perform serial blood gas measurements intraoperatively. Maintenance of $\mathrm{PaO}_{2}$ should be kept at a higher normal range in the perioperative period. Intraoperative parameters signifying bullae rupture include a progressive desaturation, diminished air entry on chest auscultation, cyanosis, tachycardia, and hypotension ${ }^{11}$. If possible, an intraoperative chest skiagram should be performed in a suspected case of pneumothorax. However, for a pulmonary emergency, emergent chest decompression followed by tube thoracostomy and chest drain should be performed on the suspected side of the chest [11]. In the advent of complications, the surgical procedure should be abandoned as soon as possible, with the return of spontaneous ventilation.

At completion of the surgery, the patient should be extubated smoothly after complete reversal of neuromuscular blockade in the postoperative care unit. In the post-operative period, adequate oxygenation, temperature control, and a multimodal analgesic approach play an important role in final patient outcome. Peri-Extubation bronchodilator treatment is also helpful. Early ambulation and chest physiotherapy should always be encouraged [12]. The patients should be meticulously monitored to avoid the risk of respiratory failure or postoperative pulmonary infections.

In conclusion, though a patient with bilateral pulmonary bullae carries a significant risk of perioperative complications, especially during positive 


\section{Anaesthesia \& Critical Care Medicine Journal}

pressure ventilation, a meticulous preoperative workup, proper planning, and vigilant perioperative team management can make the task hassle-free for the anaesthesiologist.

\section{References}

1. Fishman A, Elias J, Fishman J, Grippi M, Senior R, et al. (2008) Fishman's Pulmonary Diseases and Disorders $4^{\text {th }}$ (Edn.), Pennsylvania: McGraw-Hill, pp: 914-918.

2. Ting EY, Klopstoclz R, Lyons HA (1963) Mechanical properties of pulmonary cysts and bullae. Am Rev Respir Dis 87: 538-544.

3. Miller, Ronald D (1991) Miller's Anesthesia $8^{\text {th }}$ (Edn.), Philadelphia, PA: Saunders/Elsevier.

4. Conacher ID(1997) Anesthesia for the surgery of emphysema. Br J Anesth 79: 530-538.

5. Eagle C, Tang T (1995) Anesthetic management of a patient with a descending thoracic aortic aneurysm and severe bilateral bullous pulmonary disease. Can J Anesth 42(2): 168-172.

6. Rozet I, Vavilala MS (2007) Risks and benefits of patient positioning during neurosurgical care. Anesthesiol Clin 25(3): 631-653.
7. Lumb A, Biercamp C (2014) Chronic obstructive pulmonary disease and anesthesia. Contin Educ Anesth Crit Care Pain 14(1): 1-5.

8. Sudaman D, Morais RJ (1990) Anesthesia for bilateral pulmonary bullae. J Cardiothorac Anesth 4(6): 778780 .

9. Bansal S, Surve RM, Venkatapara RJ (2014) Anesthetic Management of a Paraparetic Patient with Multiple Lung Bullae. J Neurosurg Anesthesiol 26(1): 85-86.

10. Sahoo RK, Nair AS, Kulkarni V, Mudunuri R (2015) Anesthetic consideration in a patient with gaint bilateral lung bullae with severe respiratory compromise. Saudi J Anaesth 9(4): 493-495.

11. Caseby NG (1981) Anesthesia for the patient with a coincidental giant lung bulla: a case report. Canad Anaesth Soc 28(3): 272-276.

12. Sahajanandan R, Singh G, Ponniah M (2008) Anesthesia for lung volume reduction surgery in bilateral bullous lung disease: A Case Report. Indian J Anaesth 52(5): 584. 